Rev. Elev. Méd. vét. Pays trop., 1977, 30 (3) : 293-302.

\title{
Elevage de Biomphalaria glabrata, Say, au Laboratoire
}

\author{
par M. GRABER $\left({ }^{*}\right)$, J. EUZEBY et J. GEVREY
}

(avec la collaboration technique de $\mathbf{M}^{\mathrm{me}} \mathrm{R}$. THOMASSET)

\begin{abstract}
RESUMÉ
Les auteurs décrivent une méthode d'élevage de Biomphalaria glabrata, Say, au laboratoire, méthode nécessitant l'emploi successif et, dans des conditions qui sont bien précisées, de bacs de ponte et de bacs de croissance.

Bien que concernant un Planorbe hôte intermédiaire de Schistosoma mansoni, agent de la bilharziose intestinale humaine, les renseignements fournis présentent un certain intérêt en médecine vétérinaire, car ils peuvent, dans leur ensemble, être transposés à d'autres mollusques vecteurs de Trématodoses animales (Fasciolose, paramphistomose, bllharzioses à Schistosoma bovis et à Schistosoma mattheei) : Limnea natalensis, Biomphalaria pfeifferi, Bulins appartenant aux genres Physopsis, Bulinus s. str. (toute la série polyploïde) et Pyrgophysa.

En outre, lorsque les conditions climatiques s'y prêtent, cette méthode, simple et pratique, permet de disposer constamment, et quelle que soit la saison, d'importantes populations de Biomphalaria glabrata destinées à diverses recherches d'ordre général, notamment des essais de molluscicides et la lutte biologique contre les mollusques vecteurs, recherches qui intéressent à la fois la médecine humaine et la médecine vétérinaire tropicale notamment.
\end{abstract}

Dans le cadre d'un contrat D. G. R. S. T. concernant la lutte biologique contre Biomphalaria glabrata, Say, vecteur à la Guadeloupe de la bilharziose humaine à Schistosoma mansoni, le Laboratoire de Parasitologie de l'Ecole Nationale Vétérinaire de Lyon a été chargé plus spécialement d'étudier l'action prédatrice des crustacés et de certains poissons vivant dans les régions tropicales.

Avant d'entreprendre quoi que ce soit, il importait de créer un élevage de mollusques capable de fournir en permanence des individus de toute taille et de tout âge.

Cet élevage, en outre, devait être simple,

Contrat D. G. R. S. T. : Equilibres et luttes biologiques.

$\left(^{*}\right)$ Chaire de Parasitologie de l'Ecole Nationale Vétérinaire de Lyon, Marcy l'Etoile, 69260 Charbonnières les Bains. peu onéreux et immobiliser le moins possible le personnel chargé de sa surveillance.

Les résultats obtenus étant susceptibles d'intéresser les chercheurs aux prises avec les mêmes problèmes, il a paru utile de les rapporter ici.

\section{MATERIEL ET METHODE}

Il existe actuellement de nombreuses méthodes d'élevage mettant en jeu des techniques différentes :

- Grands bacs de 7501 reproduisant 1e plus fidèlement possible le milieu aquatique naturel. Ils sont disposés à l'extérieur sous un simple auvent et sont exposés aux vents dominants (1).

- Locaux fermés, spécialement aménagés à l'extérieur du laboratoire pour abriter des 
bacs de grande capacité, de 110 à 6501 (13, 28).

- Aquarium de moins de 501 ( (5 à 45 l) recevant $(16,22,23)$ ou non $(3,4,20,29)$ un équipement plus ou moins sophistiqué. Ces récipients, en général peu encombrants, sont placés sur des tables, sur des étagères accrochées aux murs ou dans des armoires ouvertes exposées à la lumière.

Compte tenu des conditions climatiques régnant dans la région lyonnaise et des moyens financiers mis à notre disposition, il n'était pas question d'utiliser l'une ou l'autre des deux premières techniques.

Aussi, dans l'enceinte même du laboratoire, une pièce spéciale a-t-elle été aménagée. Mesurant $40 \mathrm{~m}^{2}$, elle est orientée Nord-Sud et reçoit la lumière grâce à une grande fenêtre donnant côté Nord. Côté Sud, l'éclairement est assuré par une porte constamment ouverte sur un petit bureau très ensoleillé en toutes saisons.

Un chauffage central par radiateur permet de maintenir une température supétieure à $20{ }^{\circ} \mathrm{C}$ de novembre à avril.

A l'intérieur de la salle d'élevage, sont disposées trois rangées parallèles de tables supportant des aquariums de verre à monture métallique, d'une capacité allant de 20 à $170 \mathrm{l}$. Une plaque de verre montée sur supports de caoutchouc les protège de la poussière.

La température de l'eau est fonction du milieu ambiant. Lorsqu'elle a tendance à baisser à l'automne ou au début du printemps, on immerge dans chaque bac une résistance électrique durant quelques heures. De cette façon, la température moyenne journalière demeure comprise entre 22 et $23^{\circ} \mathrm{C}$ de janvier à mai et entre 21 et $23{ }^{\circ} \mathrm{C}$ de septembre à décembre. Durant l'été, les fenêtres de la salle d'élevage demeurant ouvertes, elle oscille entre 22 et $25^{\circ} \mathrm{C}$.

L'eau utilisée est celle du robinet. Comme elle renferme un peu de chlore, on la neutralise à la Contra-Chlorine.

Un filtre aspirateur « Ehcim» à circulation continue et pourvu d'un filtre à charbon de bois permet de purifier l'eau qui est rejetée dans le bac à l'aide d'une rampe percée de trous, ce qui assure la bonne oxygénation du milieu. Quand les mollusques sont nombreux dans le bac, on ajoute deux pompes de type
«Belbul munies chacune d'un tuyau de plastique terminé par un «sucre» qui plonge jusqu'au fond du récipient.

Le bac est nettoyé toutes les fois que l'eau se charge trop abondamment de matières organiques en décomposition (cadavres de mollusques en particulier), c'est-à-dire, selon l'importance des populations et la saison, tous les deux ou trois mois. L'eau est filtrée sur gaze et sur tamis à mailles très fines, de manière à recueillir le plus grand nombre possible de jeunes mollusques. On évite d'ailleurs, autant que faire se peut, de procéder à cette opération lorsque les jeunes, nouvellement éclos, sont nombreux dans l'aquarium. Des feuilles de laitue crue sont distribuées tous les 2 ou 3 jours. Les autres variétés de salades, les scaroles d'hiver notamment, sont moins bien consommées : elle ne le sont que dans la mesure où elles parviennent à se décomposer partiellement dans l'eau, ce qui demande un certain temps.

\section{RESULTATS}

Deux cent quarante Biomphalaria glabrata adultes ont été ramenés de Guadeloupe en décembre 1972. Ils ont été immédiatement répartis dans deux bacs de 1701 et l'évolution de la population a été suivie sur une période de 13 mois.

En mai 1973, il existait dans les aquariums 2770 individus de toute taille, soit 11,5 fois plus que la population initiale.

Cette population est alors dédoublée dans deux autres bacs de 1701 et dans un bac de 901.

En octobre 1973, 8220 mollusques ont été dénombrés dans les cinq aquariums en service, soit 34,2 fois plus qu'en décembre 1972 et, en février 1974, 9060 individus, soit 37,7 fois plus.

En 13 mois, chaque Biomphalaria a donné naissance à 36 individus nouveaux.

Quelques observations ont été faites sur la reproduction de Biomphalaria glabrata au laboratoire.

Les mollusques, après autofécondation ou accouplement, pondent sur les surfaces lisses de l'aquarium, de préférence dans les zones 
les moins exposées à la lumière, c'est-à-dire dans les angles. La plupart du temps, les pontes sont déposées sur les parties hautes, à partir de $15-20 \mathrm{~cm}$ du fond. D'un diamètre variant entre 4 et $8 \mathrm{~mm}$, elles sont plates, ovalaires, jaunâtres et renferment un nombre variable d'cufs serrés les uns contre les autres, par rangs de 1 à 4 . Elles sont entourées d'une capsule élastique résistante, ce qui permet de les détacher facilement de la paroi.

Dans un aquarium où est rassemblée une importante population (800 à 1000$)$ d'âge égal, le nombre de pontes augmente régulièrement pendant 12 jours avant d'atteindre un pallier et cette période de ponte maximale dure une semaine. Puis, le nombre de pontes émises diminue progressivement durant une douzaine de jours. Ultérieurement, une nouvelle population en âge de se reproduire s'ajoutant à la population initiale, le pallier de ponte maximale a tendance à s'allonger (10-12 jours).

Le développement des œufs est sujet à d'amples variations : ainsi 20 Biomphalaria adultes de $18 \mathrm{~mm}$ de diamètre sont placés dans un aquarium de 201 le 29-4-1974. Ils se mettent à pondre aussitôt, la température étant constamment maintenue entre 22 et $23^{\circ} \mathrm{C}$. Les pontes éclosent en 7-10 jours et de nombreux jeunes Biomphalaria sont visibles sur les parois du récipient. On retire alors les adultes. Un mois après l'éclosion, les jeunes sont déposés dans un autre aquarium. Ils grossissent peu à peu et se mettent à pondre à partir du 12 juillet.

D'œuf à æuf, il s'écoule donc un peu plus de deux mois. Dans ces conditions, il est possible d'obtenir chaque année six générations de Biomphalaria, ce qui confirme les observations de BRUMPT (3).

Toutefois ce n'est pas une règle générale et, dans certains cas, ce laps de temps peut être réduit à quatre ou cinq semaines : c'est ce que nous avons observé à trois reprises au cours des mois d'août 1974, 1975 et 1976.

\section{COMMENTAIRES}

Par cette méthode, on obtient, au mieux 10000 mollusques par an, ce qui s'est révélé suffisant pour mener à bien les essais de lutte biologique entrepris en 1974 et 1975 avec l'écrevisse américaine, Cambarus affinis.
Toutefois, dans cet élevage, le rendement œufs/mollusques s'avère extrêmement faible, sans aucune mesure avec les excellents résultats enregistrés ailleurs (24).

On sait, en effet, que la longévité d'un Biomphalaria (*) est d'environ 15-18 mois et qu'il est capable d'émettre chaque mois 20 pontes de 50-80 cufs, soit un total de 14 à 18000 œufs $(3,10)$ durant toute son existence. Le pourcentage d'éclosion dépasse 80 p. 100. Dans ces conditions, le nombre de mollusques comptés à la fin de la période d'observation de 13 mois aurait dû être beaucoup plus élevé.

Aussi, avons-nous cherché à mieux préciser les facteurs susceptibles d'influer sur les élevages de mollusques au laboratoire. Malgré toutes les recherches entreprises $(3,4,5,6$, $7,8,9,24,25$ ), on les connaît encore assez mal. Ce sont :

\section{DES FACTEURS ABIOTIQUES}

\subsection{La température}

On admet que, pour tous les mollusques vecteurs, la température moyenne optimale de l'eau se situe entre 20 et $26^{\circ} \mathrm{C}$.

Pour Bulinus (Physopsis) globosus et pour Bulinus truncatus, la reproduction est parfaitement assurée entre 20 et $25^{\circ} \mathrm{C}$, l'optimum se situant autour de $25^{\circ} \mathrm{C}$.

Pour Biomphalaria pfeifferi, le développement des populations est rapide à $25^{\circ} \mathrm{C}$. Il baisse à $19^{\circ} \mathrm{C}$. Il est très faible à $30^{\circ} \mathrm{C}$. Sur le terrain, ce mollusque ne semble pas supporter des températures supérieures à $28^{\circ} \mathrm{C}$, ce qui, dans les régions chaudes et sèches d'Afrique tropicale, limite son aire d'implantation.

Biomphalaria glabrata $(3,26)$ résiste à des températures plus élevées de l'ordre de 35$37^{\circ} \mathrm{C}$, à condition qu'elles ne soient pas constantes. A $35^{\circ} \mathrm{C}$, les mollusques cessent de se multiplier. A $30^{\circ} \mathrm{C}$, la reproduction est fortement perturbée : les pontes sont peu nombreuses mais les éclosions s'effectuent rapidement et la croissance des jeunes est accélérée.

(*) Lorsque l'eau des bacs est trop riche en matières organiques, la longévité est ramenée à moins de 12 mois. 


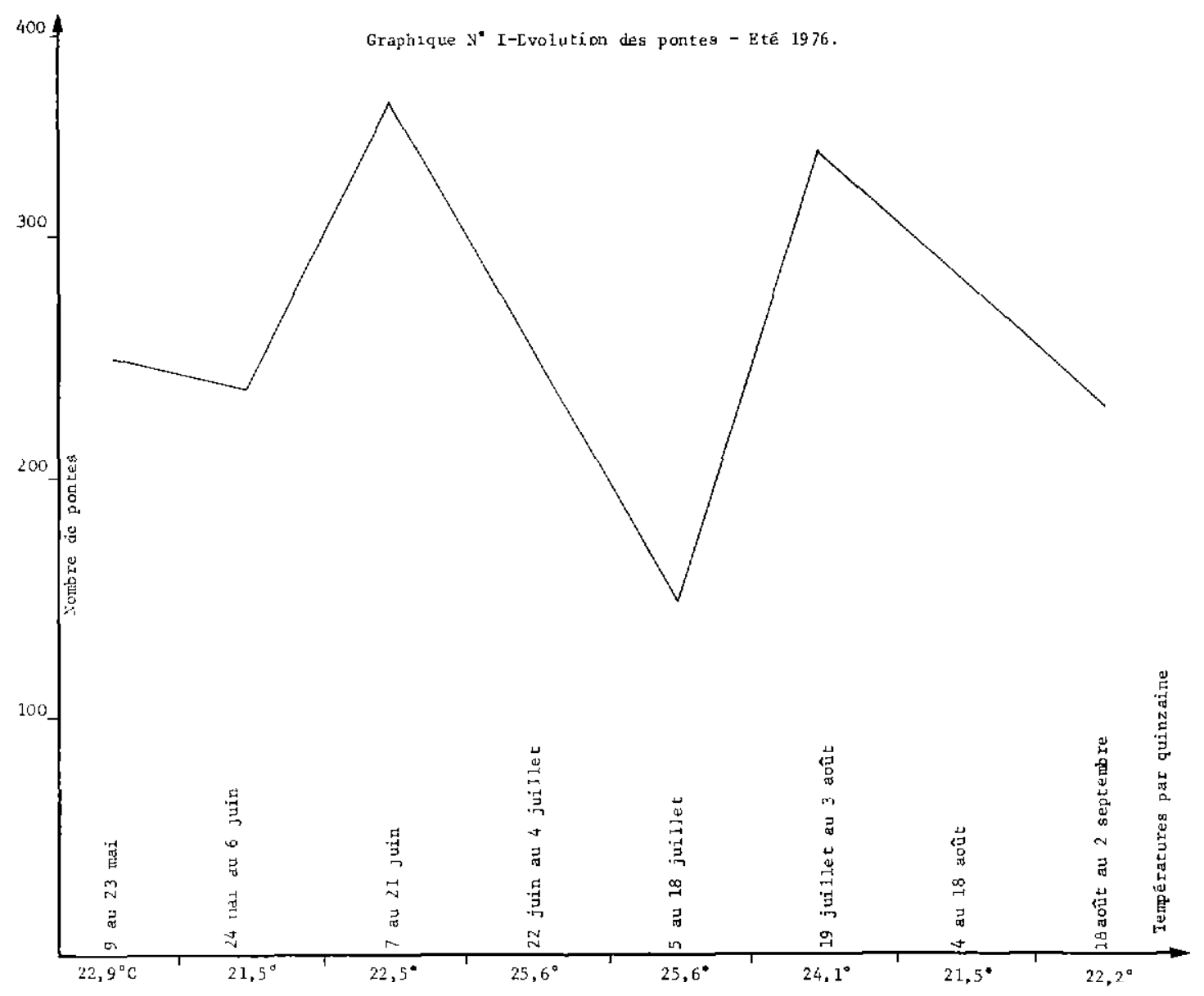

A $15-20^{\circ} \mathrm{C}$, les mollusques s'alimentent mal et se reproduisent difficilement. Le développement des jeunes est fortement ralenti.

Les températures élevées régnant dans la région lyonnaise à la fin du printemps et au début de l'été 1976 ont permis de faire quelques observations intéressantes. Les pontes déposées par des Biomphalaria (1000 individus environ) dans les angles de deux grands aquariums ont été comptées régulièrement une fois par semaine et la température journalière de l'eau soigneusement relevée. Des moyennes ont été établies par quinzaine. Les résultats figurent au graphique $\mathrm{n}$ " 1 .

A partir du 15 juin, la température des deux bacs augmente brutalement passant de $22{ }^{\circ} \mathrm{C}$ à $25^{\circ} \mathrm{C}$, pour atteindre $27^{\circ} \mathrm{C}$ entre le 2 et le 7 juillet, puis elle se maintient autour de $26^{\circ} \mathrm{C}$ jusqu'au 19 juillet. On assiste, durant cette période, à une diminution du nombre de pontes de l'ordre de 50 p. 100 . A $27^{\circ} \mathrm{C}$, un certain nombre de Biomphalaria adultes meurent et on retrouve leurs coquilles vides flottant à la surface de l'eau.

A partir du 20 juillet, la température baisse et le nombre de pontes remonte sensiblement pour revenir peu à peu au niveau atteint à la fin mai-début juin.

Il apparaît donc qu'une température dépassant régulièrement les $25^{\circ} \mathrm{C}$ pendant plus d'un mois est déjà néfaste à la reproduction des mollusques, alors que normalement (infra, graphique $\mathrm{n}^{\circ} 2$ ), à cette période de l'année et pour une température de $23-25^{\circ} \mathrm{C}$, le nombre de pontes est maximal.

Il est donc inutile de maintenir dans les aquariums une température trop élevée et, dans les conditions de notre élevage, elle se situe, selon la saison, entre 21 et $25^{\circ} \mathrm{C}$.

\subsection{La lumière}

Son rôle est encore mal connu (24). Classiquement, on considère que la lumière inter- 
vient de façon indirecte sur la croissance de la flore et de la faune nécessaires à la nourriture des mollusques.

Récemment, des essais effectués par JOY (14) ont apporté quelques données nouvelles. $\mathrm{La}$ lumière n'a pratiquement pas d'influence sur la croissance des Biomphalaria. En revanche, les rayons infra-rouges augmentent le nombre de pontes nocturnes, si le temps d'exposition est de 12-14 heures de lumière et de 1012 heures d'obscurité. Les pontes diminuent pour 14 heures de lumière et 10 heures d'obscurité.

\subsection{Le Ph}

Le rôle du $\mathrm{Ph}$ est négligeable, les mollusques supportant, en effet, des $\mathrm{Ph}$ compris entre 4,8 et 9,8. Le $\mathrm{Ph}$ de l'eau utilisée à Lyon est voisin de la neutralité $(7,4)$.

\subsection{Minéralisation}

Au laboratoire, la minéralisation n'intervient que comme facteur alimentaire dans l'élaboration de la coquille. Les besoins des Biomphalaria sont de 0,25 g p. 1000 de chlorure de sodium et de 0,05 g p. 1000 de phosphate de calcium.

Dans les eaux non minéralisées, l'élevage des Planorbes est impossible et la carence des eaux en calcaire se traduit par des dystrophies de la coquille sous forme de plaques érosives blanchâtres, nacrées et situées, en général, au centre de celle-ci. Bien que l'eau distribuée dans la région lyonnaise soit fortement chargée en sels de calcium, des érosions de ce type ont été observées à plusieurs reprises dans les bacs d'élevage, mais elles ne sont pas constantes et touchent les individus les plus âgés.

Pour vérifier l'hypothèse d'un manque de calcium, plusieurs centaines de jeunes mollusques ont été placés dans un aquarium rempli d'eau provenant d'une source connue pour être particulièrement riche en sels de calcium dissous. Au bout de 6 mois, on note sur les exemplaires les plus gros l'apparition de taches érosives, comme avec l'eau du robinet. Peutêtre, faut-il admettre avec BRUMPT (3) que les corrosions de la coquille sont liées aux *habitudes grégaires des Biomphalaria, les individus de cette espèce pouvant, dans cer- taines circonstances $\left(^{*}\right)$, s'agglomérer les uns aux autres et attaquer les coquilles de leurs congénères » qui, une fois atteintes, sont envahies plus facilement par les algues, des champignons ou des bactéries.

1.5. L'oxygénation, à partir des filtres aspirateurs "Eheim», est suffisante, les besoins en oxygène des mollusques vecteurs étant relativement faibles (7).

On a préconisé, pour assurer une bonne oxygénation, l'introduction dans les aquariums de diverses plantes aquatiques : Vallisneria spiralis, Sagittaria, Elodea canadensis, Myriophyllum japonicum et surtout Ludwigia palustris $(18,24,25)$.

En réalité, ces plantes servent principalement à recueillir les pontes qui ne sont alors plus décrochées par les mollusques en mouvement sur les parois de l'aquarium, comme cela arrive assez souvent.

La présence de plantes aquatiques ne peut se concevoir que dans la mesure où une nourriture artificielle est donnée aux mollusques, le grand développement qu'elles prennent souvent gênant la distribution en quantité suffisante des feuilles de laitue à la surface des bacs.

1.6. La richesse de l'eau en matières organiques joue également un rôle important. La limite de tolérance des mollusques vecteurs est très grande, surtout lorsqu'il s'agit de matières organiques d'origine végétale. A titre d'exemple, il a été trouvé, en février 1974, dans des bacs où vivaient, depuis plus de deux mois, d'importantes colonies de mollusques, les quantités suivantes (**):

- Bulinus sp. (Ethiopie) : 7,2 mg/1.

- Limnaea natalensis (Tchad, Ethiopie) : $6,2 \mathrm{mg} / \mathrm{l}$.

\section{- Bromphalaria pfeifferi (Ethiopie) $6,2 \mathrm{mg} / 1$.}

- Biomphalaria glabrata (Guadeloupe) : $6,8 \mathrm{mg} / 1$.

(*) Lorsque les bacs sont très riches en matières organiques, que les salades en cours de décomposition s'accumulent dans les angles ou, quand au moment du nettoyage des aquariums, on remplace l'eau à $23^{\circ} \mathrm{C}$ par de l'eau a $16-17^{\circ} \mathrm{C}$

(**) Résultats exprimés en mg d'oxygène nécessaire pour oxyder les matières organiques contenues dans un litre d'eau. Celle-ci est considérée comme polluée à partir de $4 \mathrm{mg} / \mathrm{l}$. 
Après nettoyage et vidange des bacs, à partir de populations de même importance, on observe un mois plus tard :

- Biomphalaria glabrata : 5,6 mg/1.

- Biomphalaria pfeifferi : $4,5 \mathrm{mg} / 1$.

- Limnaea natalensis : $2,3 \mathrm{mg} / 1$.

$\mathrm{Ce}$ sont les Biomphalaria morts, beaucoup plus que leurs déjections, qui provoquent la pollution accélérée de l'eau. Il importe donc de surveiller attentivement les élevages et d'éliminer systématiquement tous les cadavres qui, avant de tomber au fond du récipient, flottent pendant quelque temps à la surface.

D'ailleurs, en cas de pollution trop accusée, les mollusques ont tendance à se regrouper à la surface et à quitter le bac.

Comme il a été dit plus haut, les bacs de 90 et 1701 sont nettoyés tous les 2 ou 3 mois et les bacs de $20 \mathrm{I}$ tous les 15 jours. Pour apprécier le degré de pollution, le meilleur test est celui de la couleur de l'eau : lorsqu'elle prend une coloration brunâtre ( comme du purin $»$ ), et que les pontes dans les angles deviennent invisibles, tant à la lumière du jour qu'à la lumière électrique, il est urgent de changer l'eau.

Parfois, dans l'aquarium, il se produit un trouble passager sous forme d'un voile verdâtre : il n'a aucune signification (22).

\subsection{La profondeur de l'eau}

Elle influe sur le comportement et la survie de jeunes Biomphalaria de moins de 15 jours (25). Leurs besoins respiratoires sont supérieurs à ceux des adultes et, de ce fait, ils sont obligés de remonter fréquemment à la surface. Ils ne peuvent descendre au-delà de $20 \mathrm{~cm}$, sinon ils périssent d'asphyxie.

\section{DES FACTEURS BIOTIQUES}

\subsection{L'alimentation}

«Les mollusques vecteurs sont des phytophages dans le cadre d'un régime alimentaire omnivore» (7). Ils se contentent de ce qu'ils trouvent : phanérogames, cryptogames, mousses, lichens, algues, flore microscopique.

Au laboratoire, on utilise, la plupart du temps, des feuilles de laitue crues ou cuites (*), les premières étant, en général, préférées aux secondes (5). Les Biomphalaria sont friands de cette nourriture qu'ils consomment continuellement et en grande quantité, en moyenne le quart de leur poids en $24 \mathrm{~h}$ (7).

On peut également distribuer d'autres aliments d'origine végétale : orties, feuilles de chou-fleur cuites, germes de blé chauffés à $120^{\circ} \mathrm{C}$, pousses d'endives crues, cresson de fontaine, feuilles d'épinard ébouillantées, ainsi que, dans les pays tropicaux, des feuilles cuites d'Eichhornia crassipes et d'Ipomea reptans. Les matières organiques en décomposition (feuilles d'arbres, bois) sont aussi très appréciées.

A certaines époques de l'année (hiver), l'administration de laitue devient très onéreuse et, pour diminuer les coûts, on a cherché à la remplacer par des aliments artificiels renfermant surtout des protides et des vitamines.

L'aliment de STANDEN (25) est à base de germe de blé, de laitue séchée en poudre, de poudre de lait et d'alginate de sodium. Le mélange subit une préparation spéciale et se présente sous l'aspect de feuilles minces qui restent à la surface de l'eau et sont, de ce fait, accessibles aux jeunes mollusques.

MOORE et collab. (18) remplacent la laitue par des algues et ajoutent une petite quantité d'une préparation commerciale destinée à la nourriture des poissons d'aquarium.

A l'alginate de sodium, ERIKSON et collab. (12) substituent du talc, car, selon eux, la présence d'alginate provoque une diminution de la ponte, des retards de croissance chez les jeunes et une moindre résistance des coquilles

D'autres mélanges ont été préconisés à base de provendes pour rats, pour chiens, pour cobayes et pour poissons.

Ces aliments sont distribués chaque jour en une ou deux fois, selon la taille des mollusques et l'importance des populations vivant dans les aquariums. Les quantités réparties doivent être rigoureusement dosées, sinon on risque de polluer le bac. Comme ce n'est guère facile, on préfère compléter cette nourriture par des

(*) Selon certains auteurs, les Biomphalaria nourris exclusivement sur laitue pondent moins d'ceufs que ceux qui reçoivent des aliments concentrés. 
feuilles de laitue qui servent alors d'aliment d'appoint (20).

Quel que soit le mode d'alimentation (laitue ou aliments concentrés), le régime doit comporter également de petits organismes vivants, notamment des algues, qui, dans les aquariums, se développent abondamment d'avril à octobre. La présence d'algues vertes est absolument nécessaire à la croissance des jeunes, les 15 premiers jours de leur existence surtout. Ce problème a retenu l'attention de tous ceux qui ont à leur charge un élevage de mollusques et différentes solutions ont été proposées pour assurer un apport maximal d'algues microscopiques : cultures d'Oscillatoria dans les récipiens où vivent les Planorbes ou adjonction de poudre d'algues en hiver quand la reproduction de ces organismes cesse, ce qui est le cas à Lyon.

\subsection{Fluctuation saisonnière des popula- tions}

Les pontes accrochées aux parois du bac (surfaces planes et angles) ont été comptées chaque jour dans un aquarium de $170 \mathrm{I}$ ensemencé avec 125 Biomphalaria glabrata. Les moyennes mensuelles ont été établies et reproduites sur le graphique $n^{0} 2$.
Dans cet élevage, le rythme de reproduction est bien marqué : faible durant l'automne et l'hiver, le nombre de pontes journalières s'accroît sensiblement à partir d'avril. Il est en mai 3,6 fois supérieur à ce qu'il était en février-mars et il se maintient à un niveau élevé jusqu'à la mi-septembre-début octobre.

$\mathrm{Au}$ laboratoire, la reproduction des Biomphalaria s'étale donc sur toute l'année avec un maximum estival et un minimum hivernal.

Cette situation n'est guère favorable, car la production de mollusques est discontinue et, en raison du «trou » hivernal, certains essais de lutte biologique qui mettent en jeu un nombre élevé de mollusques (de 1000 à 1500 ) risquent, de février à juin, d'être fortement ralentis, voire interrompus.

\subsection{La surpopulation}

Dans le même bac d'élevage qui a servi aux observations précédentes, ont été décomptés :

- Au départ, le 7-1-1973, 125 Biomphalaria adultes.

- Le 15-5-1973 (décompte $n^{\circ}$ 1), 500 individus, soit 4 fois plus.

- Le 15-10-1973 (décompte $\mathrm{n}^{\circ}$ 2),

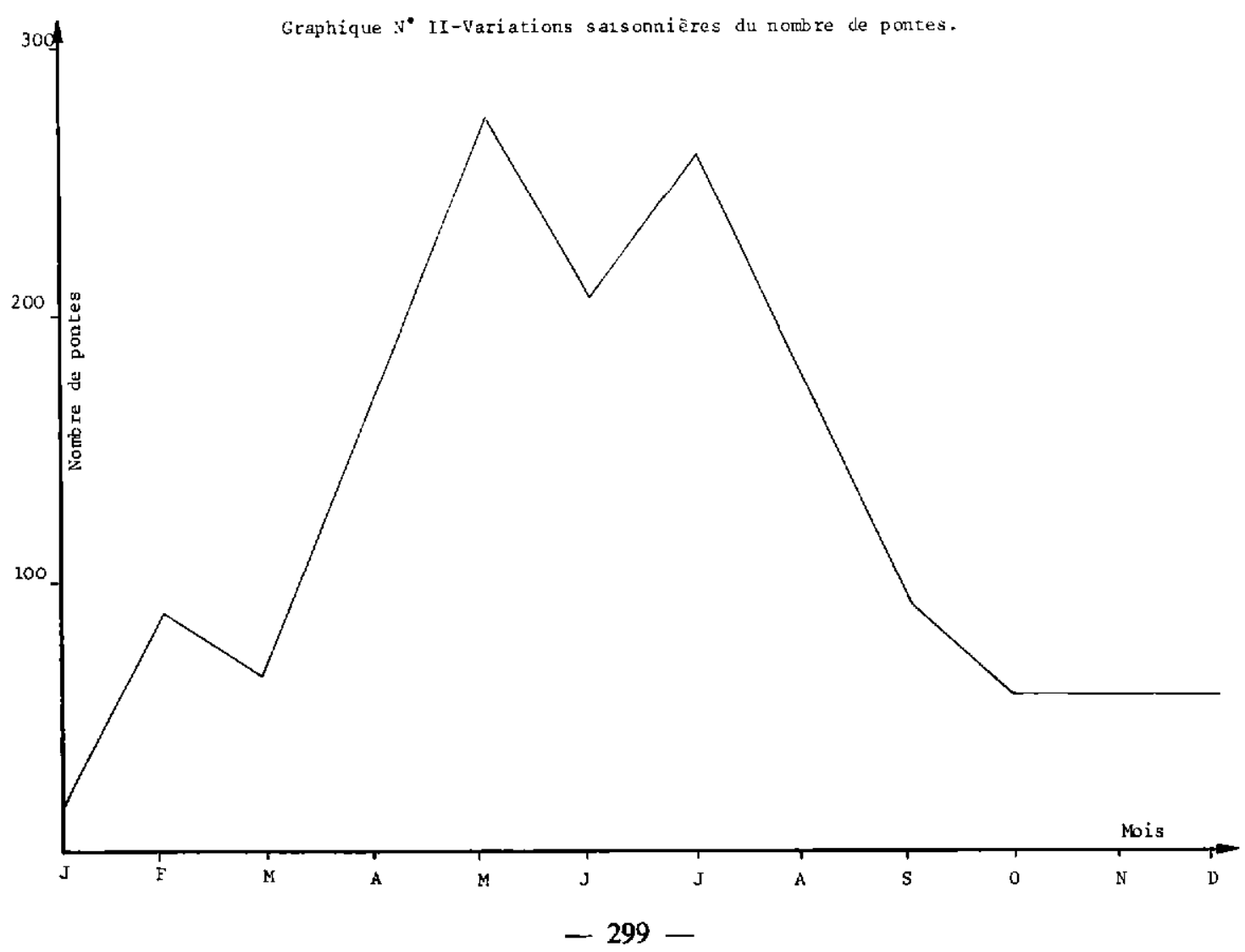


2230 mollusques, soit 17,8 fois plus. La mortalité est de 84 individus (les plus âgés), soit 3,9 p. 100.

- Le 31-1-1974 (décompte $\mathrm{n}^{\circ} \quad 3$ ), 2500 Biomphalaria, soit 25 fois plus. La mortalité est de 326 individus, soit 13 p. 100.

Entre le décompte 1 et le décompte 2, la population est multipliée par 4,4 et, entre le décompte 2 et le décompte 3, par 1,1 .

La population du bac qui s'est accrue très rapidement et dans les mêmes proportions entre janvier et mai et entre mai et octobre, demeure stagnante entre octobre et février.

Outre la diminution de nombre de pontes joumalières au cours de la période hivernale (voir supra), il faut incriminer également la surpopulation de l'aquarium. Connu depuis le milieu du siècle dernier sous le nom d'\& Overcrowding effect », ce phénomène a été décrit à de nombreuses reprises $(2,3,4,10,27,30)$.

Dans les bacs d'élevage, l'effet de surpopulation se traduit par une diminution sensible de la fécondité des mollusques portant à la fois sur le nombre global d'œufs et sur le nombre d'œufs par ponte et par un ralentissement de la croissance des jeunes avec des coquilles de moindre taille.

En outre, les pontes déposées sur les parois du récipient peuvent être, avant éclosion, décrochées par les adultes en surnombre qui sont capables, dans certains cas, de dévorer les jeunes nouvellement éclos.

Plusieurs théories ont été proposées pour expliquer ce phénomène : rythmes biologiques fortement perturbés avec comme conséquences modifications profondes des habitudes alimentaires et des dépenses d'énergie des mollusques (4) ; présence dans l'eau des aquariums d'une phéromone ou de diverses substances toxiques provenant de la décomposition de la nourriture ou des déjections, substances qui ne sont, d'ailleurs, pas stables et qui peuvent être éliminées en changeant l'eau des bacs régulièrement (30) : manque d'oxygène et alimentation insuffisante $(10,20)$; épuisement de l'oxygène dissous dans une eau qui n'est pas suffisamment renouvelée.

En réalité, des études récentes (27) montrent que l'effet de surpopulation joue aussi lorsqu'un petit nombre de mollusques est entretenu dans un espace restreint et que, pour se multiplier et se développer normalement, le mollusque doit disposer d'un volume d'eau minimal et d'une surface convenable.

Pour Bulinus tropicus et Bulinus truncatus, on estime le volume à $100 \mathrm{ml}$ par mollusque, un peu moins pour Bulinus forskalii. Pour les Planorbidae, de plus grande taille comme Biomphalaria glabrata, les meilleurs résultats ont été obtenus avec $200 \mathrm{ml}$ par individu $(2,21)$.

\section{MODIFICATIONS APPORTEES A LA TECHNIQUE D'ELEVAGE}

Compte tenu de toutes ces observations, il est apparu nécessaire, au cours de l'automne 1974, d'apporter à la méthode d'élevage un certain nombre de modifications en utilisant des bacs de ponte et des bacs de croissance dans les conditions suivantes :

1) Dans des cristallisoirs de verre de 15-20 1, on dépose 15 Biomphalaria de 15 à $22 \mathrm{~mm}$ de diamètre. Les bacs exposés à la lumière sont régulièrement chauffés et la température de l'eau oscille entre 22 et $23^{\circ} \mathrm{C}$. De la salade fraîche est distribuée en abondance et, deux fois par semaine, on ajoute une pincée d'algues en poudre.

Les mollusques qui jouissent ainsi de conditions de température et d'alimentation optimales se mettent à pondre rapidement et massivement. Une dizaine de jours plus tard, quand le nombre de pontes sur les parois atteint 150 et avant que les œufs n'éclosent, on retire les Biomphalaria et on nettoie le récipient.

Les pontes évoluent peu à peu. Elles donnent naissance à des jeunes qui ne sont plus gênés par les adultes et trouvent dans le milieu les algues nécessaires à leur développement. La faible hauteur des cristallisoirs $(15 \mathrm{~cm})$ leur permet, en outre, de venir respirer à la surface plus facilement que dans les aquariums de plus grande capacité. De cette façon, les pertes sont minimes.

L'eau est changée toutes les 2 ou 3 semaines.

Selon les besoins et les possibilités de stockage, on ensemence un nombre plus ou moins important de bacs.

2) Dès que les coquilles mesurent $5 \mathrm{~mm}$ de diamètre, les Biomphalaria sont mis dans des aquariums de 90 et 1701 , à raison de 450-500 individus dans le premier cas et de 850-900 dans le second : on évite ainsi l'effet de surpopulation. La nourriture est à base de 
poudre pour poissons et de salade fraîche à volonté. On immerge, selon la capacité des bacs, un ou deux bâtons de craie. La température est maintenue régulièrement entre 22 et $25^{\circ} \mathrm{C}$. Le nettoyage a lieu tous les deux mois : dans l'intervalle on aspire le plus gros des déjections à l'aide d'un tuyau de plastique que l'on promène doucement au fond de l'aquarium.

Ce système inspiré de méthodes couramment utilisées dans les laboratoires se livrant à l'élevage des mollusques vecteurs $(18,22,23)$ permet de disposer :

- Dans les grands aquariums, de réserve d'environ 4500 à 5000 individus pouvant être immédiatement utilisés dans les essais de lutte biologique.

- Dans les cristallisoirs, d'un nombre de jeunes suffisant pour remplacer, dans un délai très court, les populations précédentes et en assurer ainsi le renouvellement régulier, quelle que soit la saison.

\section{CONCLUSIONS}

L'élevage de Biomphalaria glabrata, Say au laboratoire ne présente pas de difficultés insurmontables, à condition de respecter certaines règles et d'assurer au mieux les besoins essentiels des mollusques.

Cependant, lorsque des populations importantes comportant des individus d'âge différent sont entretenues dans des bacs de 90-170 l, le rendement des élevages n'est pas toujours satisfaisant. Trois facteurs limitants interviennent : la surpopulation, le rythme de reproduction des Planorbes, maximal en été, minimal en hiver et la mortalité élevée des jeunes nouvellement éclos.

Pour éviter ces inconvénients, il est recommandé :

- de retirer les adultes après le dépôt des pontes, de faire éclore séparément les jeunes dans des baos de 201 bien aérés et chauffés régulièrement et de leur fournir en abondance une nourriture à base d'algues en poudre ;

- de stocker les individus de plus de $5 \mathrm{~mm}$ de diamètre, ainsi que les adultes, dans des aquariums de plus grande capacité, à raison de 450-500 individus pour 901 d'eau et de $850-900$ pour 170 l. Ils seront entretenus selon les règles habituelles en prenant soin, toutefois, de compléter l'alimentation à base de feuilles de laitue fraîche par une distribution régulière de provende pour poissons.

\section{SUMMARY}

Maintenance and breeding of Biomphalaria glabrata, Say, in laboratory

A method is described for laboratory culture of Biomphalaria glabrata, Say, the intermediate host of Schistosoma mansoni.

The adult and young mollusc's requirements for maintenance in captivity are discussed (aquaria, aeration, light, feeding, water pollution).

The authors study the overcrowding phenomenon and the seasonal variations of Biomphalaria glabrata populations characterized by a high rate of reproduction in summer and a low one in winter.

To avoid these restricting factors, they propose a single and practical solution.

\section{RESUMEN}

\section{Crianza de Biomphalaria glabrata Say, en laboratorio}

Los autores describen un método de crianza de Biomphalaria glabrata Say en laboratorio, método necesitando el empleo sucesivo, y en condiciones bien precisadas, de cubetas de postura y de cubetas de crecimiento.

Aunque concerniendo un huesped intermediario de Schistosoma mansoni, agente de la bilharziosis intestinal humana, las informaciones obtenidas presentan un cierto interés en medicina veterinaria, porque pueden, en su conjunto, ser transpuestas a otros moluscos vectores de trematodosis animales (Fasciolosis, paramfistomosis, bilharziosis con Schistosoma bovis y Schistosoma mattheei) Limnaea natalensis, Biomphalaria pfesfferi, bulinos perteneciendo a los generos Physopsis, Bulinus s. str. (toda la serie poliploida) y Pyrgophysa.

Además, cuando las condıciones climáticas son favorables, dicho método, simple y práctica, permite tener constantemente, cualquiera que sea la estación, importantes poblaciones de Biomphalaria glabrata destinadas a varias investigaciones generales, particularmente los ensayos de belicidos y la lucha biológica contra los moluscos vectores, investigaciones que interesan a la vez la medicina humana y la medicina veterinaria tropical. 


\section{BIBLIOGRAPHIE}

1. BIRGI (E.), GRABER (M.). Mollusques pulmonés d'eau douce basommatophores vecteurs au Tchad des affections parasitaires du bétail. Possibilıtés d'élevage au Laboratoire. Rev. Elev. Méd. vét. Pays trop., 1969, 22 (3) ; 393-408.

2. BRENES (E.), RADKE (M. G.), RITCHIE (L. S.). Effects of crowding on $A$. glabratus as observed under laboratory conditions. $J$. Irop. Med. Hyg., 1958, 7 (2) 242.

3. BRUMPT (E.). Mission E. BRUMPT et L. Ch. BRUMPT au Vénézuela. Observations biologiques diverses concernant Planorbis (Australorbis) glabratus, hôte intermédiajre de Schistosoma mansoni. Annls. Parasit. hum. comp., 1941, 18 (1/3) : 9-45.

4. CHERNIN (E.), MICHELSON (H.). Studies on the biological control of Schistosoma-bearıng snails. III. The effects of population density on growth and fecundity of A. glabratus. Am. J. Hyg., 1957, 65 (1) : $57-70$.

5. COWPER (S. G.). Some notes on the maintenance and breeding of Schistosoma vectors in Great Britain, with special reference to Planorbis guadeloupensis. Ann. trop. Med. Parasit., 1946, 40 (2): 163-170.

6. DESCHIENS (R.). Les facteurs conditionnant l'habitat des mollusques vecteurs de bilharzioses. Leurs incidences épidémiologiques. I. : Généralités, facteurs physiques. Annls. Inst. Pasteur, Paris, 1957, 92 (5) : 576-585.

7. DESCHIENS (R.). Les facteurs conditionnant l'habitat des mollusques vecteurs de bilharzioses. Leurs incidences épidémiologiques. II. : Facteurs chimiques. Nutrition. Annls Inst. Pasteur, Parts, 1957, 92 (5) : 711-727.

8. DESCHIENS (R.). Les facteurs conditionnant l'habitat des mollusques vecteurs de bilharzioses. Leurs incidences épidémiologiques. III. : Flore et faune des gîtes, ennemis naturels, maladies, compétitions, fluctuations. Annls. Inst. Pasteur, Paris, 1957, 93 (1) : 1-12.

9. DESCHIENS (R.). Les facteurs conditionnant l'habitat des mollusques vecteurs de bilharzioses. Leurs incidences épıdémiologiques. IV. Constantes physiologiques de la reproduction et particularités en fonction des espèces. Conclusions. Ann/s. Inst. Pasteur, Paris, 1957, 93 (2) : 153-167.

10. ETGES (F. J.). Laboratory studies on the effect of crowding upon fecundity of $A$. glabratus. Parasitology, 1963, 49 (5, Sect. 2) : 24-25.

11. ETGES (F. J.), RITCHIE (L. S.). Comparative observations on growth rate and reproduction of A. glabratus in field and laboratory conditions. Bull. Wld. Hlth. Org., 1966, 34 (6) : 963-966.

12. ERIKSON (D. G.), RITCHIE (L. S.), CALDWELL (D. R.). Growth and reproduction of $A$. glabratus fed alginated food. Parasitology, 1961, 47 (4, Sect. 2) : 50 .

(*) Une Bibliographie beaucoup plus complète est à la disposition des lecteurs intéressés qui pourront l'obtenir gratuitement en s'adressant au Centre de Documentation de l'I. E. M. V. T., 10, rue Pierre-Curie, 94700 MaisonsAlfort, France.
13. GRETILLAT (S.). Rapport I. E. M. V. T.-Laboratoire Elevage Dakar Hann, Sénégal, 1964, 17 p.

14. JOY (J.E.). The influence of day length upon the egg-laying of Biomphalaria glabrata. Ann. trop. Med. Parasit., 1971, 65 (4) : 573-578.

15. LAGRANGE (E.). Fécondité et régime d'Australorbis glabratus. Bull. Soc. Path. exot, 1957, 50 (5) 804-811.

16. LEE (C. L.), LEWERT (R. M.). The maintenance of Schistosoma mansoni in the laboratory. J. infect. Dis., 1956,99 (1) : 1-14.

17. MICHELSON (E. H.) The effects of temperature on growth and reproduction of Australorbis glabratus in the laboratory. Am. J. Hyg., 1961. 73 (1): 66-74.

18. MOORE (D. V.), THILLET (C. J.), CARNET (D. M.), MELENEY (H. E.). Experimental infection of Bulinus truncatus with Schistosoma haematobium. Parasitology, 1953, 39 (2) : 215-221.

19. RIPSON (C. A.). Reduction of the time factor in rearing Australorbis glabratus. Am. Midl. Nat., 1949, 42 (3) : 757-758.

20. RITCHIE (L. S.), BERRIOS-DURAN (L. A.), DEWEESE (R.). Biological potentials of Australorbis glabratus: growth and migration. Am. J. trop. Med. Hyg., 1963, 12 (2) : 264-268.

21. RITCHIE (L. S.), HERNANDEZ (A.), ROSAAMADOR (R.). Biological potentials of Biomphalaria glabrata: life-span and reproduction. Am. J. trop. Med. Hyg., 1966, 15 (4) : 614-617.

22. ROWAN (W. B.). Mass cultivation of Australorbis glabratus intermediate host of Schistosoma mansoni in Puerto-Rico. Parasitology, 1958, 44 (2) : 247.

23. SANDT (D. G.), BRUCE (J. I.), RADKE (M. G.). A snail colony facility for the mass production of Schistosoma mansoni cercariae. Parasitology, 1965, 51 (6) : 1012-1013.

24. STANDEN (O. D.). Experimental schistosomiasis. I. The culture of snall vectors Planorbis boissyi and Bulinus truncatus. Ann. trop. Med. Parasit., 1949, 43 (1) : 13-22.

25. STANDEN (O. D.). Some observations upon the maintenance of Australorbis glabratus in the laboratory. Ann. trop. Med. Parasit, 1951, 45 (1) : 80-83.

26. STURROCK (R.), STURROCK (B. M.). The influence of temperature on the biology of Biomphalaria glabrata, Say, intermediate host of Schistosoma mansoni in St Lucia, West Indies. Ann. trop. Med. Parasit., 1972, 66 (3) : 385-390.

27. STURROCK R,), STURROCK (B. M.). Observations on some factors affecting the growth rate and fecundity of Biomphalaria glabrata. Ann. trop. Med. Parasit., 1970, 64 (3) : 349-355.

28. SWART (P. J.), REINECKE (R. K.). Studies on Paramphistomiasis. I. The propagation of Bulinus tropicus Krauss, 1848. Onderstepoort $J$. vet. Res., 1962, 29 (2) : 183-187.

29. WARD (P. A.), TRAVIS (D.), RUE (R. E.). Methods of establishing and maintening snails in the laboratory. Fed. Sec. Agency, U.S. Publ. Hith. Serv., Nat. Inst. Hith. Bull., 1947 (189) : 70-80.

30. WRIGHT (C. A.). The crowding phenomenon on laboratory colonies of freshwater snails. Ann. trop. Med. Parasit., 1960, 54 (2) : 224-232. 